\title{
Maximum energy conversion from human motion using piezoelectric flex transducer: A multi-level surrogate modeling strategy
}

\author{
Liheng Luo ${ }^{1}$, Dianzi Liu', Meiling Zhu', Yijie Liư ${ }^{4}$, and Jianqiao Ye ${ }^{1}$ \\ ${ }^{1}$ Department of Engineering, Lancaster University, Lancaster, UK \\ ${ }^{2}$ Faculty of Science, University of East Anglia, Norwich, UK \\ ${ }^{3}$ College of Engineering, Mathematics and Physical Sciences, University of Exeter, Exeter, UK \\ ${ }^{4}$ Department of Engineering Mechanics, Guangzhou University, Guangzhou, PR China
}

\begin{abstract}
Conventional engineering design optimization requires a large amount of expensive experimental tests from prototypes or computer simulations, which may result in an inefficient and unaffordable design process. In order to overcome these disadvantages, a surrogate model may be used to replace the prototype tests. To construct a surrogate model of sufficient accuracy from limited number of tests/simulations, a multi-level surrogate modeling strategy is introduced in this paper. First, a chosen number of points determined by Optimal Latin Hypercube (OLH) Design of Experiments are used to generate global-level surrogate models with Genetic Programming and the fitness landscape can be explored by Genetic Algorithms for near-optimal solutions. Local-level surrogate models are constructed then from the extended-OLH samples in the vicinity of global optimum on the basis of a much smaller number of chosen points. As a result, an improved optimal design is achieved. The efficiency of this strategy is demonstrated by the parametric optimization design of a Piezoelectric Flex Transducer (PFT) energy harvester. The optimal design is verified by finite element simulations and the results show that the proposed multi-level surrogate modeling strategy has the advantages of faster convergence and more efficiency in comparison with the conventional single level surrogate modeling technique.
\end{abstract}

Corresponding authors:

E-mail address: dianzi.liu@uea.ac.uk (D.Liu); j.ye2@lancaster.ac.uk (J. Ye) 


\section{Keywords}

Multi-level optimization strategy, Surrogate model, Energy harvesting, Design of Experiments, Genetic Programming, Piezoelectric flex transducer

\section{Introduction}

Traditional engineering design processes normally require a large number of expensive experimental tests from prototypes which may be unaffordable and ineffective. Benefiting from the rapid development of computer-aided design engineering, finite element analysis (FEA), which can numerically predict the performance of a design by virtual computer experiments, has become a popular method to replace a traditional design process so that the cost of experimental tests can be greatly reduced. Andrade et al. (2008) developed a finite element model of an acoustic levitator and used the model to determine the optimal geometry of the device. The optimal design of the acoustic levitator was verified by experimental results. Olayan et al. (2012) performed finite element (FE) simulations of thin-film, multi-layer piezoelectric pressure sensor in order to obtain maximum deflection and voltage generation of the piezoelectric layer. Leinonen et al. (2014) developed a FE model to consider the electro-mechanical coupling effect of a piezoelectric cymbal harvester. The results suggested the FE model was able to predict power output of the cymbal harvester with an acceptable error of 7\%. Tabatabaei et al. (2016) optimized the shape of a piezoelectric cantilevered energy harvester using analytical formulation and the optimal design was verified by the results 
obtained from FEM simulations. However, due to the increase of design complexities, a large number of combinations of design variables have to be considered in order to find the optimal design in the entire design space. This is prohibitively expensive, as each simulation with fine meshes to be examined in use of finite element method may be computationally intensive. At this point, surrogate models are often employed to replace the original FE simulations so that the design optimization problem can be solved efficiently by using mathematical algorithms. Marcelin (2004) proposed a numerical optimization approach for car gear box mechanism by using neural networks surrogate model. Kim et al. (2016) optimized a piezoelectric cantilever beam energy harvester by introducing kriging surrogate model to approximate the relationship between the design variables and the responses predicted by the FE model, and Halder and Samad (2017) optimized the geometric parameters to improve the performance of wave energy harvesting turbines by employing also Kriging surrogate model.

In order to construct an efficient surrogate model, Design of Experiment (DoE) sampling techniques, such as factorial designs, central composite design (CCD), orthogonal arrays, Latin hypercube, etc., are usually applied to achieve a uniform distribution of sampling points in the design space. Since the number of combinations of design variables grows exponentially as the number of design variables increases, building a high-fidelity surrogate model to represent a complex FE model or a physical test is still a time-consuming process. To further reduce the computational cost of surrogate modeling, advanced techniques for fast convergence of solutions were 
proposed. To this end, Siah et al. (2004) proposed a fast optimization technique that used hybridized Kriging surrogate model and Divided Rectangles (DIRECT) optimization algorithm to find the global optimum efficiently. Forrester et al. (2009) demonstrated a multi-fidelity optimization for aircraft wing drag pressure via coKriging surrogate model, by which the surrogate model was constructed to enhance the low-fidelity Kriging approximation. Sun et al. (2011) presented a two-stage multifidelity Response Surface (RS) model based on the ratio between two models with different fidelity. This modeling method was then used to optimize the draw-bead design in sheet-metal forming process to improve computational efficiency. Viana et al. (2013) developed a multiple surrogate efficient global optimization (MSEGO) algorithm which is able to generate multiple sampling points in each cycle to improve the efficiency of surrogate-based optimization. Demonstrated by optimizing an antenna design, Liu et al. (2016) proposed a multi-fidelity surrogate modeling framework to improve the efficiency of multi-fidelity surrogate model assisted optimization. More recently, Vincenzi and Gambarelli (2017) improved the efficiency and accuracy of surrogate-assisted evolutionary algorithm by combining it with a proper infill sampling strategy and a quadratic surrogate approximation method. Li et al. (2017) proposed a multi-fidelity surrogate modeling method with Cooperative Radial Basis Function (CoRBF), which showed improved efficiency, accuracy and robustness when compared to the RBF and co-Kriging methods.

This paper introduces a multi-level surrogate model optimization scheme integrated 
with the optimal Latin hypercube Design of Experiments (OLH DoE) sampling technique for optimal design of a piezoelectric energy-harvesting device. The optimization process has two stages. In the first stage, a global-level surrogate model is built by Genetic Programming (GP) to seek the maximum power output of the PFT device subject to displacement and strength constraints. The OLH DoE is used to determine the distribution of sampling points for the surrogate modelling. In the second stage, a refined design space is defined in the vicinity of the optimum achieved previously and a local-level surrogate model is developed to find a better design with maximum power output. In this multi-level surrogate model optimization scheme, Genetic Algorithm (GA) is applied to find the global optimum. Finally, the potential of the developed multi-level surrogate modelling strategy for solving complex design problems is demonstrated by parametric optimization of the PFT energy harvesting device. The results show that the power generated by the optimal design has been increased by over $200 \%$ when a safety factor of 1.0 is considered in the design process. Furthermore, a comparison study between the proposed multi-level surrogate modeling technique and the single level surrogate modeling technique demonstrates that the global-local surrogate model assisted optimization strategy has the power to solve complex engineering optimization problems with high efficiency and accuracy.

\section{Coupled piezoelectric finite element model (CPC-FEM)}

The development of modeling piezoelectric energy harvester has a long history (Yan, 
2018). For a better understanding of vibrating energy harvesters, Sodano et al. (2004) developed a distributed parameter model for cantilevered piezoelectric energy harvester based on the Rayleigh-Ritz piezoelectric actuator model (Hagood et al., 1990) to estimate the power output. Recently, Yoon et al. (2018) studied time-varying performances of piezoelectric vibration energy harvesting devices under nonstationary random vibrations. Zhu et al. (2009) developed a coupled piezoelectric-circuit finite element (CPC-FE) model for cantilevered piezoelectric energy harvester to study the performance of the harvester under different load frequencies and resistance load. The FE model considered the electro-mechanical coupling effect and was applied to compute power output. The CPC-FE model for the cymbal type piezoelectric energy harvester was implemented in ANSYS by Daniels et al. (2013) to analyze the mechanical-electrical interaction of a piezoelectric actuator (Newnham et al., 2000). The accuracy of predictions using the CPC-FE model for the coupled analysis was validated against the experimental results. Base on the study on the CPC-FE model for the cymbal design, a Piezoelectric Flex Transducer (PFT) that is capable of withstanding a larger magnitude of force was proposed. The PFT has a rectangular shape as shown in Figure 1 and the values of its initial design parameters were selected based on the cymbal device design. The PFT was manufactured and experimentally tested, the results of which were used for validation of FE models, including the one used in this paper. Luo et al. (2017) successfully applied the metamodeling technique to obtain an optimal design of the PFT device by parametric optimization. The optimal design offered a power output of $6.54 \mathrm{~mW}$, representing an improvement of more than 
$58 \%$ as compared to the power output of $4.13 \mathrm{~mW}$ from the original PFT design when a safety factor of 2.0 is applied.

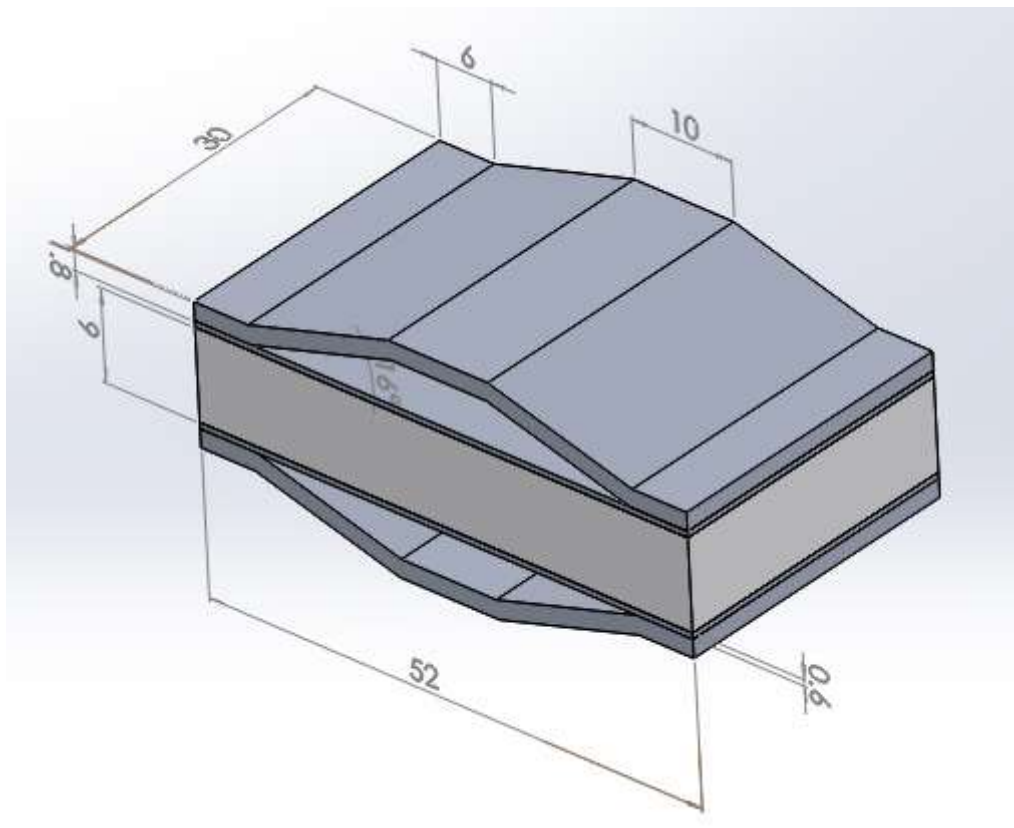

Figure 1. An optimized Piezoelectric Flex Transducer (PFT) device

\section{Multi-level surrogate modeling strategy}

A complex engineering design usually requires solving a parametric optimization problem, which is constrained by a set of bounded design variables to seek the optimal solution. Without the help from a proper parametric optimization technique, these design variables keep changing within their allowable bounds so that an infinite number of combinations of parameters have to be enumerated to represent all the designs in the entire design space. Obviously, this process is computationally prohibitive. Parametric optimization techniques tackle this problem by examining an affordable number of designs to predict the optimal solution and also provide an explicit function of parameters for the prediction of the optimal values. However, the accuracy of the 
explicit function or surrogate model strongly depends on the Design of Experiments (DoE) sampling technique and the size of the samples. Forrester et al. (2009) reviewed the infill sampling technique and described its basic procedure driven by the Radial Basis Function (RBF) for efficiently descending to a local minimum instead of the global minimum, as shown in Figure 2(a). This is due to the low fidelity of the approximate surrogate model. To reduce the computational cost of surrogate modelling while maintaining its high fidelity, the sampling points in this paper are determined by the OLH DoE technique for a uniform distribution across the entire design space so that the surrogate models can be used as an approximation to the physical model and predict the system responses with sufficient accuracy.

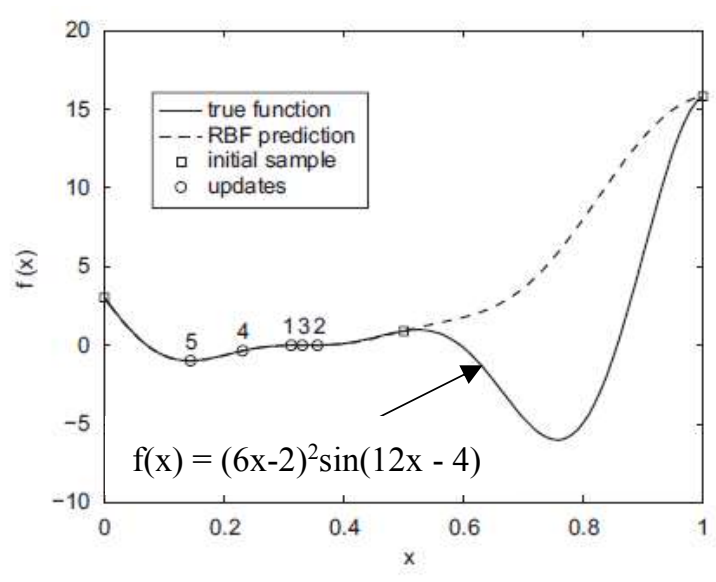

(a)

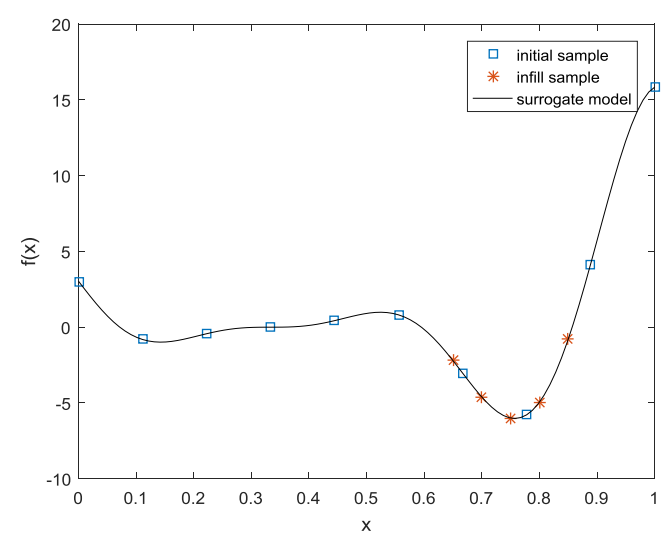

(b)

Figure 2. (a) Surrogate model with infill points strategy descending into a local optimum (Forrester et al., 2009), (b) An improved approximation by multi-level surrogate modelling strategy 
On the basis of the above, a multi-level surrogate modeling method with the OLH DoE sampling technique is developed in this paper to avoid falling into local optimums and efficiently seek the global optimum. This strategy includes two stages: i.e., the globallevel exploration and the local-level exploitations. The global-level exploration aims to find the promising design space where the global optimum lies in, while the purpose of the local-level exploitation is to increase the accuracy of the surrogate model within the promising subset using the additional sampling points in the refined design space. The search for the near-optimal solution in the global-level exploration stage starts with applying the OLH DoE technique to generate uniformly distributed sampling points in the entire design space. The developed CPC-FEM is used then to run the coupled mechanical-electrical simulations at these design points. Given the training data, the global-level surrogate models are built by Genetic Programming (GP). Next, GA is applied to the global-level surrogate models to explore the design space for the nearoptimal solution. In the local-level exploitation stage, extra sampling points are generated by extended OLH DoE in the vicinity of the near-optimal design space, which is determined in the global-level exploration. Then, the new surrogate model with higher accuracy is generated to find the optimal design within this promising design space. Finally, the optimal design is validated by FE simulations. If the predictions by the surrogate models do not agree with the simulation results within the acceptable tolerance, the surrogate models will be rebuilt using more sampling points in the local exploitation stage and the optimization will be executed with a higher accuracy surrogate model until the optimal solution is found. 
The efficiency of the proposed multi-level surrogate modeling strategy with the infill sampling points selected by the OLH DoE technique is demonstrated by the illustrative function described in Figure 2(a). Ten optimized initial sampling points by OLH are first used to explore the global design space. The predicted minimum value of the global-level surrogate model is around $\mathrm{x}=0.8$, as shown in Figure 2(b). Following that, additional five infill sampling points are uniformly located in the predicted optimum design space to update the surrogate model. Then, the gradient based optimization is performed on the updated local-level surrogate model. The flowchart of the multi-level surrogate modeling strategy employed in this paper is shown in Figure 3. The main process of the multi-level surrogate modeling and parametric optimization employed to maximize the power output of the PFT device is explained as follows:

First, 140 uniformly distributed initial sampling points are selected by the optimal Latin Hypercube DoE technique within the global design space. These sampling points representing designs of 7 design variables are analyzed by FE simulations to obtain the outputs of the system. Then, these training data are used to construct global level surrogate models by GP to find the near-optimal solution. Subsequently, 30 additional sampling points are selected in the near-optimal vicinity to build the local level surrogate model for exploiting the optimal solution in the local design space. Finally, FE simulation, which was validated by experimental results, is performed to validate the optimal solution predicted by the surrogate models. 


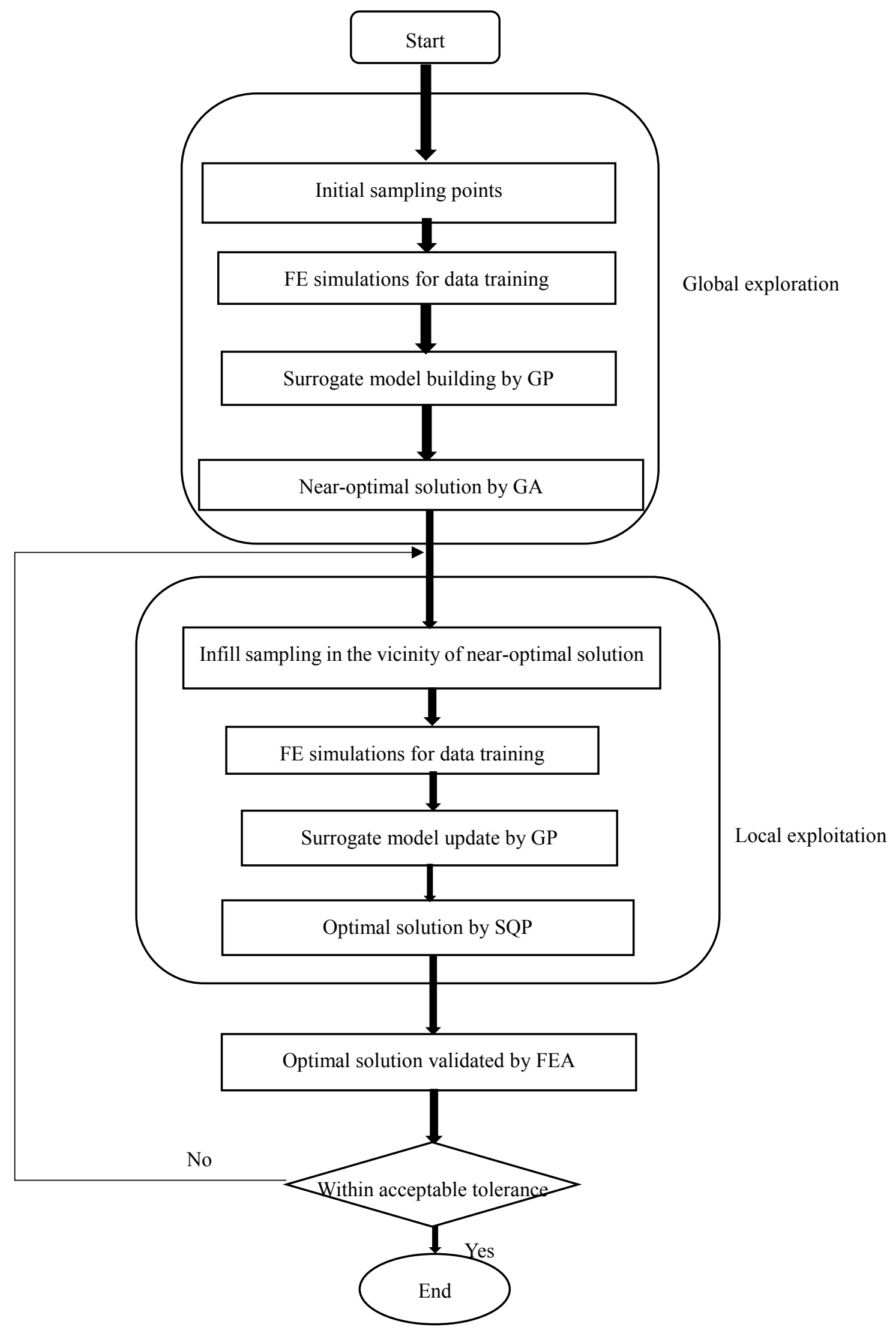

Figure 3. Flowchart of the multi-level surrogate modeling strategy with OLH

sampling technique 
The detailed design of the PFT device is a multi-parameter optimization problem, for which the multi-level surrogate models are constructed to seek the maximal power output. As mentioned above, the proposed multi-level optimization strategy consists of the global exploration and the local exploitation stages, each of which includes independent modelling, optimization and FE validation process. Summarily, the strategy comprises four main steps:

I. Design of Experiments (DoE)

II. Surrogate model building by Genetic Programming (GP)

III. Surrogate model assisted optimization

IV. Optimal results and validation by FE simulations

In the following Sections, details of the four steps will be introduced.

\section{Design of Experiments (DoE)}

Conventional engineering design optimization process used to vary one parameter a time to observe the relationship between the system outputs and the varied input parameters. However, with the rapid development of manufacturing tools and advanced materials, the complexity of the design is significantly increased in order to obtain the potential benefits provided. As a consequence, the numbers of design variables are increased accordingly and solving such design problems becomes more challenging. It is evident that the conventional one-factor-at-a-time experimental method to determine the impact of two or more factors on responses of interests is less effective due to its simple 
mechanism.

Design of Experiments (DoE) is a statistical technique (Box, 1980) to simultaneously study the effect of multiple variables with high efficiency and it can successfully deal with design optimization projects of various engineering applications. Based on the sampling points recommended by the DoE technique, a statistic model, which is usually called the surrogate model, is built to replace physical experimental tests and full scale numerical simulations. Therefore, the time required for physical experiments or computer experiments is significantly reduced. Furthermore, the approximate surrogate model built by the DoE design points, where a design point is related to a specific set of design parameters, can be utilized as a relevant objective function whose optimal solution represent effectively the solution of the original complex optimization problem. A survey of different kinds of DoE is given by Simpson et al. (1997). However, the quality of a metamodel strongly depends on an appropriate choice of DoE type and the sampling size (Forrester et al., 2008). In order to explore the design space in a limited number of computer experiments and improve the efficiency of DoE, a uniform Latin hypercube DoE (Toropov et al., 2007) based on the use of the Audze-Eglais optimality criterion (Audze and Eglais, 1977), is used in this paper.

The main requirements of the optimal Latin hypercube DoE are as follows:

- The number of the set of design variables is equal to the number of experiments and for each set of the design variables there is only one experiment allowed;

- The points corresponding to the experiments are distributed as uniformly as possible in the design space where each design parameter is defined as an independent co-ordinate. Thus, the Euclidean distance between two neighboring 
points can be calculated using Pythagorean formula as follows.

$$
U=\sum_{p=1}^{P} \sum_{q=p+1}^{P} \frac{1}{L_{p q}^{2}} \rightarrow \min
$$

where, $P$ is the total number of points, $L_{p q}$ is the distance between points $p$ and $q(p \neq q)$. Minimizing $U$ produces a system (DoE) where points are distributed as uniformly as possible in the design space.

It should be noted that the above principles for the determination of sampling points are applied in both the global-level and the local-level surrogate modelling stages. In this paper, 140 uniformly distributed design points are proposed for the FE simulations for global surrogate model building. In order to reflect the influence of existing sampling points in the global-level surrogate modelling stage on the distribution of the sampling points used for the local level approximation model building, an additional requirement has to be met in the local-level surrogate modelling stage, i.e.,

- The sampling points used in the local-level surrogate model building should be determined such that the distance between any local point and the existing global point satisfies Eq. (1).

To satisfy all above requirements, the extended OLH DoE is defined.

In the local-level surrogate model building, 30 uniformly distributed design points are proposed for the FE simulations and the bar chart of the minimum distances between all 30 sampling points is shown in Figure 4, which indicates a good uniformity of sampling points by the extended OLH DoE. 
As a rule of thumb, the number of distributed design points should be at least $(\mathrm{n}+1)(\mathrm{n}+2) / 2$, where $\mathrm{n}$ is the number of design variables. In principle, the required number of sampling points increases exponentially with the increase of design parameters. The quality of surrogate models, which relies upon the number of sampling points, should be tested by the accuracy of the predictions. If the accuracy of the predictions is not satisfactory, the number of points should be increased until the predictions have good agreement with the FE simulations or experimental tests.

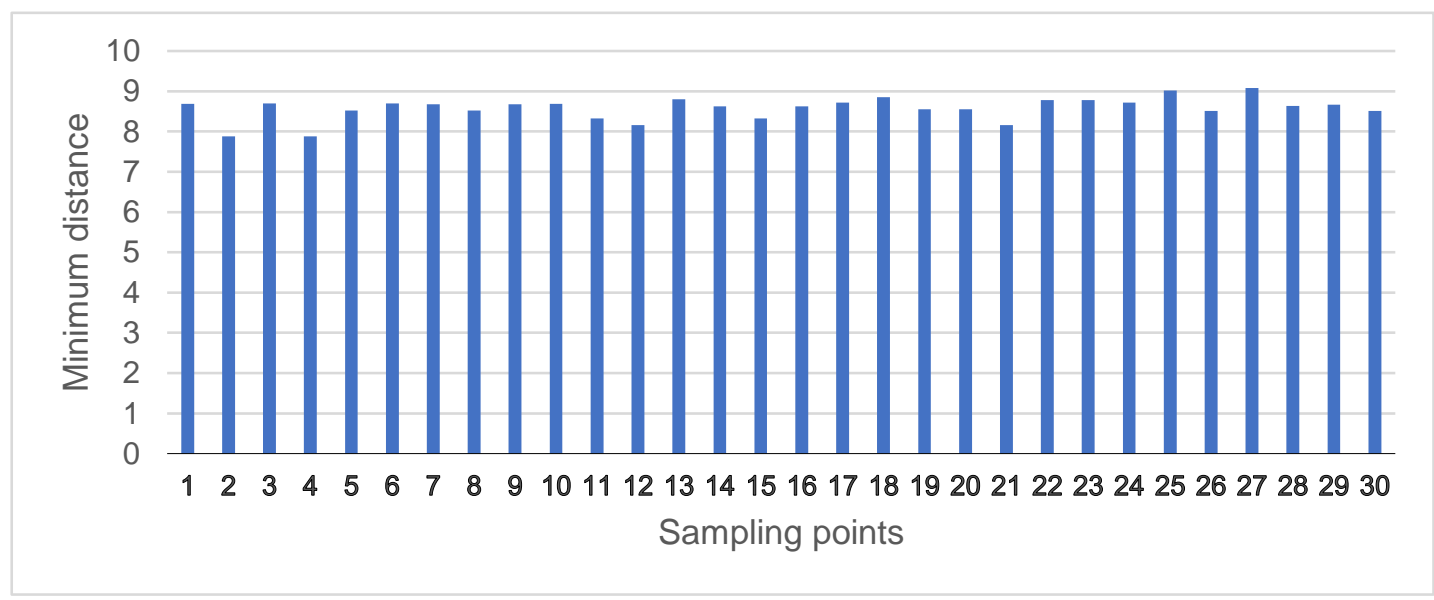

Figure 4. Minimum distances between points determined by 30-point extended OLH DoE for local-level surrogate model building

\section{Genetic Programming (GP)}

Genetic programming methodology (GP) (Armani et al., 2011; Koza, 1992) is a systematic way of selecting a structure of high quality global approximations. The GP code was first developed according to the guidelines provided by Koza (1992), then implemented for symbolic regression tasks by Armani et al. (2011). The common 
genetic operations used in GP are reproduction, mutation and crossover, which are performed on the mathematical expressions (metamodels) stripped of their corresponding numerical terminals. Parameters are inserted in the offspring and then optimized during the fitness evaluation. Selection of the structure of an analytical approximation function is a problem of empirical model building and this also results in solving a combinatorial optimization problem. Even if a bank of all regressors is established, the search through all possible combinations would result in prohibitive computational effort. In this case of design optimization, the program represents an empirical model to be used for approximation of a response function. Details and implementations of genetic programming used in this paper can be found in Armani (2014).

In order to encourage the evolution of compact, smooth, and accurate mathematical expressions and to avoid 'bloating' (Poli et al., 2008), the definition of fitness values $F(i, t)$ of individual $i$ at generation $t$ has been represented in the form of a weighted sum of different terms or objectives, as an approach to solve the multi-objective optimization using the evolution-based algorithm:

$$
F(i, t)=a_{1} F_{1}(i, t)+a_{2} F_{2}(i, t)+a_{3} 10^{6} F_{3}(i, t)+a_{4} F_{4}(i, t)
$$

where,

$$
a_{1}+a_{2}+a_{3}+a_{4}=1
$$

$F_{1}$ is the root mean square error (RMSE) of the $i$ th individual in the $t$ th generation 
evaluated on the given data set, divided by the average RMSE of the archive individuals at the previous generation; $F_{2}$ is the square of the number of numerical coefficients (parameters) present in the individual; $F_{3}$ is the number of operations not defined (i.e. division by zero) in the individual at any of the DOE sample point. F3 becomes very small when it is taken near the optimal solution. Therefore, to ensure that $F_{3}$ is evaluated numerically in a similar range to the other three objective functions, an amplification factor of $10^{6}$ is introduced before $F_{3} . F_{4}$ is the number of nodes that the individual is made of and $a_{1}, a_{2}, a_{3}$ and $a_{4}$ are weighting factors (that add up to 1 ) determined by the exhaustive testing and tuning of the GP algorithm (Armani et al., 2011). Their values were: $a_{1}=0.8989, a_{2}=0.001, a_{3}=0.1$ and $a_{4}=0.0001$ in this study. The above GP framework is built at both the global and local level to construct the respective surrogate models, which are to be optimized subsequently to predict maximum electric power output of the PFT device.

\section{Surrogate Model Assisted Optimization of the PFT Design}

The finite element method (FEM) is widely used for solving engineering design problems because it can effectively predict the performance of complex and expensive prototype experiments. In this paper, ANSYS Parametric Design Language (APDL) is applied to perform the parametric analysis of the PFT device, which is a mechanical and electrical coupling system. The basic components of the PFT are a piezoelectric plate, two substrate layers on the top and bottom of the plate covered by two metal 
endcaps placed outside the substrate layers. The geometric parameters of the PFT device and its three-dimensional finite element model are shown in Figures 2 and 5, respectively. Detailed material properties of the PFT device can be found in the work (Daniels, 2014).

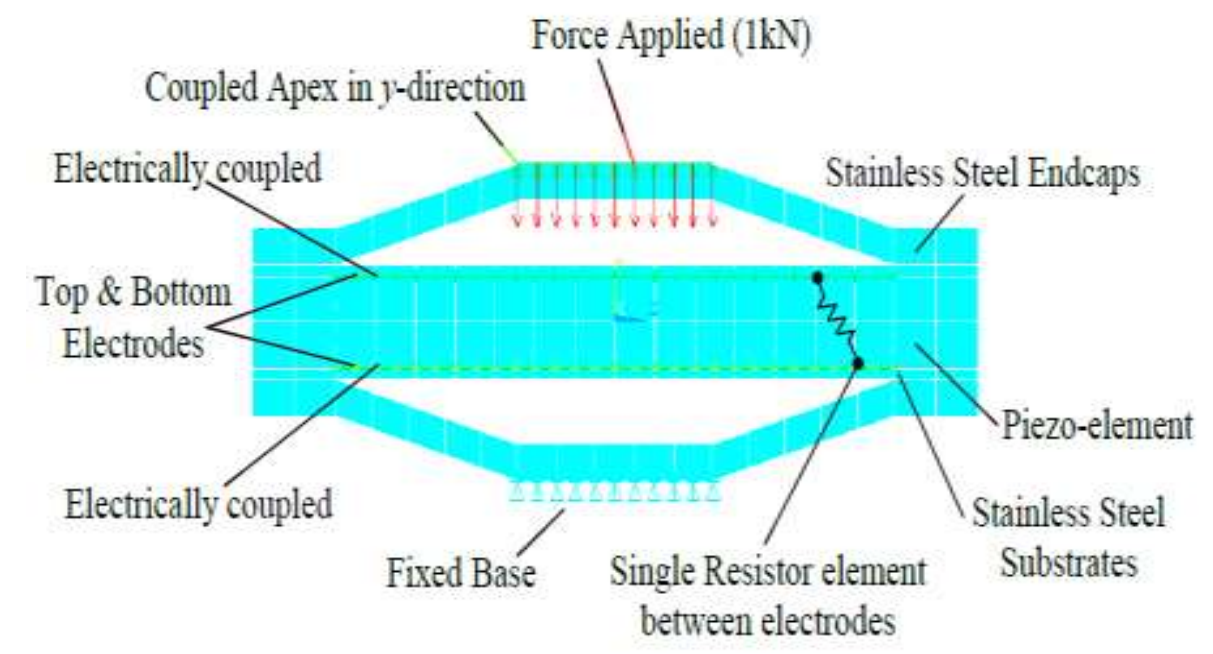

Figure 5. FE modelling of the PFT device using APDL (Daniels, 2014)

The boundary conditions of the FE model are defined as follows:

- No displacement in the vertical direction is allowed at the bottom surface of the device ;

- $\quad 1 \mathrm{kN}$ load is uniformly distributed on the top surface of the device;

- Two electrodes are located, respectively, on the top and the bottom surfaces of the piezoelectric material;

- A resistor connects the two electrodes.

To obtain the maximal power output of the PFT, seven parameters including 6 geometric parameters shown in Figure 6 and the resistant load $(R)$ are selected as design 
variables in the following optimization process. The geometric design variables are depicted as: the cavity length $\left(D_{c}\right)$, the apex length $\left(D_{a}\right)$, the endcap internal angle $(\theta)$, the respective thicknesses of the piezoelectric plate $\left(t_{p}\right)$, the substrate layer $\left(t_{s}\right)$, and the cap $\left(t_{c}\right)$. The total length $(D)$ and width $(W)$ shown in Figure 1 are constant in this study and are $52 \mathrm{~mm}$ and $30 \mathrm{~mm}$, respectively. By altering the cavity length, the internal angle, and the apex length, the overall height of the cap $(H)$ will be changed. The joint length $(J)$ represented in Figure 6 can be defined as follows:

$$
J=\frac{D-D_{C}}{2}
$$

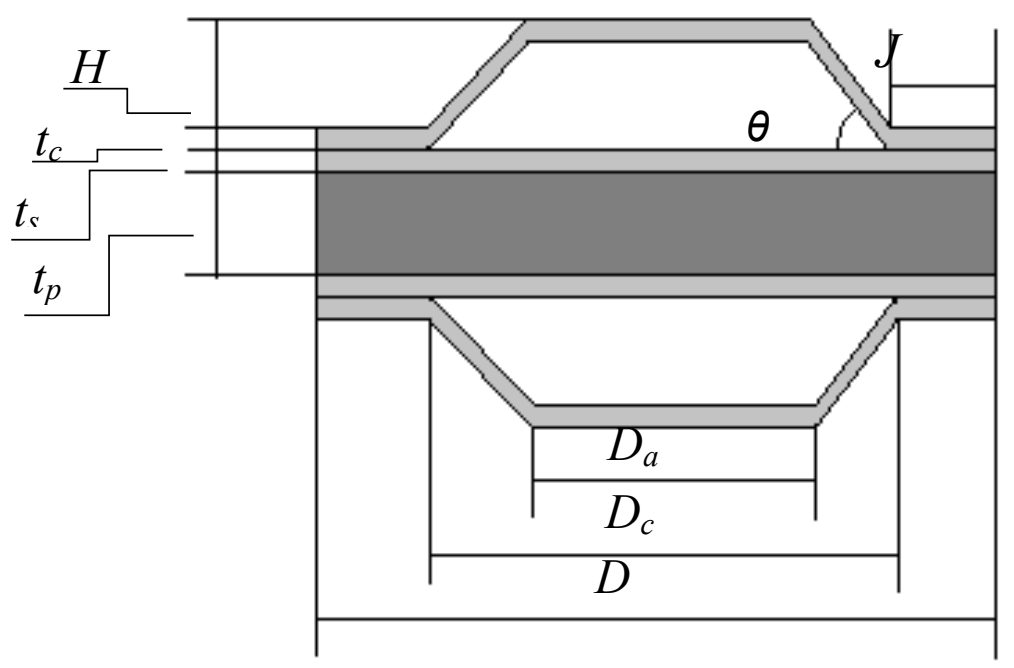

Figure 6. Design variables of PFT in parametric optimization.

The bounds of the seven design variables are listed in Table 1. These upper and lower bounds of the parameters are chosen according to the practical and manufacturing restraints. During the optimization procedure, the yield stress of material and the maximum allowable displacement are also taken into consideration. The maximum principal stress of the device is calculated using the FE model. 
Table 1. Bounds of design variables.

\begin{tabular}{|l|l|l|}
\hline DVs & Lower Bound & Upper Bound \\
\hline Thickness of PZT, $\mathrm{t}_{\mathrm{p}}(\mathrm{mm})$ & 0.5 & 9 \\
\hline Thickness of substrate, $\mathrm{t}_{\mathrm{s}}(\mathrm{mm})$ & 0.6 & 0.9 \\
\hline Thickness of endcap, $\mathrm{t}_{\mathrm{c}}(\mathrm{mm})$ & 0.5 & 4 \\
\hline Length of cavity, $\mathrm{D}_{\mathrm{c}}(\mathrm{mm})$ & 25 & 40 \\
\hline Length of apex, $\mathrm{D}_{\mathrm{a}}(\mathrm{mm})$ & 9.8 & 18.2 \\
\hline Internal angle, $\theta(\mathrm{deg})$ & 5 & 45 \\
\hline Resistive loads, $\mathrm{R}(\mathrm{M} \Omega)$ & 1 & 19 \\
\hline
\end{tabular}

The maximum principal stress, which occurs in the metal endcap, is measured during the optimization procedure and compared with the material yield stress. The design is considered a failure if the maximum principal stress is larger than the material yield stress.

The apex downward displacement should not exceed the height $H$ so that the contact between the cap and the substrate can be avoided in the process of energy harvesting. Thus, the optimization problem is formulated as below:

To find: $\operatorname{Max} P$

Subject to the bounds specified in Table 1 and:

$$
\begin{aligned}
& \frac{D_{\text {disp }}}{H}<1 \\
& \frac{\sigma}{\sigma_{y}} \leq 1
\end{aligned}
$$


where $P$ denotes the non-dimensional electrical power that is normalized by the maximum electrical power predicted from the initial 140 sampling designs before the failure criteria is applied. The maximum principle stress is normalized by the yield stress of the material and the maximum displacement of the apex is normalized by the height of the endcap, $H$. When a safety factor of 1.0 is chosen for both the displacement and stress criteria, equations (5) and (6) are the respective stiffness and strength constraints, in which the height of the endcap, $\mathrm{H}$, can be computed by

$$
H=\frac{D_{c}-D_{a}}{2} \tan \theta
$$

In Equations (5) and (6), $D_{\text {disp }}$ is the displacement of the apex; $\sigma_{y}$ is the yield stress of the endcap material; and $\sigma$ is the maximum principal stress in the endcap.

The parametric optimization of the PFT is performed using the multi-level surrogate models constructed from the OLH DoE sampling strategy, which has been introduced in the Design of Experiments Section. As described in the Multi-level surrogate modeling strategy Section, 140 initial points are taken uniformly from the entire design space for global surrogate model building and 30 extra points are sampled in the vicinity of the near-optimal design space (promising design space) for the local surrogate model building.

\section{Optimal Results and Validation by FE Simulations}

In Table 2, the optimal results obtained using global-local surrogate models and FE simulation are compared to demonstrate the validity and effectiveness of the proposed 
multi-level surrogate modeling strategy. It is noted that in the global-level exploration phase, the landscape feature of the electrical power function in the PFT device design can be approximately predicted using the low-fidelity surrogate model, which is built by 140 sampling points. Based on the surrogate models built by GP, Genetic Algorithm (GA), as a search tool, is applied on the surrogate models for seeking the near-optimal solution in the global level. Although the predicted normalized displacement agrees well with FE results, the discrepancy of $6.38 \%$ exists between the normalized maximum principal stress $(0.88)$ obtained by the global-level surrogate model and the FE validation result (0.94). In terms of the optimal electrical power, significant differences between the surrogate and FE models are observed. These differences imply that it is challenging to accurately predict the complex responses, such as electric power and stress, in use of a single level surrogate model. However, this simple surrogate modeling strategy has the advantage in describing the profile of the complex responses with reduced computational effort. Based on the optimal design by the global-level exploration, the refined design space of design variables in the near-optimal vicinity for the local level exploitation is shown in Table 3 . These updated bounds of the promising search domains are applied to perform the local level optimization for a solution with higher accuracy. By exploiting the local-level surrogate model built using extra 30 extended OLH samples, the optimal design of the PFT device for maximal electric power is finally found by the SQP technique. The last two rows in Table 2 show a maximal difference of $2 \%$ between the predicted results $(0.97)$ and the FE validation (0.99) for the normalized electric power. It is concluded that with a total number of 170 
design points, the design optimization of PFT device with 7 design variables have been solved accurately and efficiently. The predicted results agree well with the FE simulations and the normalized electric output of the optimized PFT device has been significantly increased from 0.52 to 0.99 by $45 \%$ for the stress safety factor of 1.0 . It can be seen that the proposed multi-level surrogate modeling strategy for design optimization of piezoelectric energy harvesting devices improves not only the accuracy of the predictions, but also not only the performance of the device.

Table 2. Optimal solution and its FE validation for the design with safety factor of 1.0

\begin{tabular}{|l|l|l|l|l|}
\hline & $\begin{array}{l}\text { Structural response } \\
\text { type }\end{array}$ & $\begin{array}{l}\text { Normalized } \\
\text { Electrical power }\end{array}$ & $\begin{array}{l}\text { Normalized maximum } \\
\text { principal stress }\end{array}$ & $\begin{array}{l}\text { Normalized } \\
\text { displacement }\end{array}$ \\
\hline $\begin{array}{l}\text { Global level } \\
\text { optimization }\end{array}$ & Predicted by GA & 1.79 & 0.88 & 0.04 \\
\cline { 2 - 5 } & Validation by FEA & 0.52 & 0.94 & 0.04 \\
\hline $\begin{array}{l}\text { Local level } \\
\text { optimization }\end{array}$ & Predicted by SQP & 0.97 & 0.99 & 0.04 \\
\hline
\end{tabular}

Table 3. Promising domains of design variables in the local-level optimization

\begin{tabular}{|l|l|l|l|l|l|l|l|}
\hline Design variables & $\mathrm{t}_{\mathrm{p}}(\mathrm{mm})$ & $\mathrm{t}_{\mathrm{s}}(\mathrm{mm})$ & $\mathrm{t}_{\mathrm{c}}(\mathrm{mm})$ & $\mathrm{D}_{\mathrm{c}}(\mathrm{mm})$ & $\mathrm{D}_{\mathrm{c}}(\mathrm{mm})$ & $\theta(\mathrm{deg})$ & $\mathrm{R}(\mathrm{M} \Omega)$ \\
\hline $\begin{array}{l}\text { Near optimal solution at } \\
\text { the global level } \\
\text { optimization }\end{array}$ & 9 & 0.9 & 0.5 & 40 & 10.68 & 14 & 13.5 \\
\hline $\begin{array}{l}\text { Refined bounds at the } \\
\text { local level optimization }\end{array}$ & $7-9$ & $0.6-0.9$ & $0.5-1$ & $38-40$ & $9.8-14$ & $10-15$ & $10-19$ \\
\hline
\end{tabular}

In order to demonstrate the superiority of the proposed multi-level surrogate modeling strategy over other optimization methodologies used in the previous work (Forrester et 
al., 2009; Luo et al., 2017), the optimal designs including optimal solution of the multilevel surrogate modeling strategy, the single level surrogate modeling approach, and the conventional varying one parameter a time optimization technique are listed and compared in Table 4. Where a safety factor of 2.0 is applied to the stress constraint. The first two rows represent the optimal designs obtained by the multi-level and single-level surrogate modeling assisted optimization strategies, respectively. The FE validation results are shown in the third row. The predicted normalized electric power by multilevel surrogate modeling strategy has a value of 0.39 , which has good agreement with the result of 0.38 by FE validation, while the normalized electric power ( 0.46$)$ obtained from the single level surrogate model is overestimated by $21 \%$ as compared to the FE results. This indicates that the multi-level surrogate modelling strategy is more accurate than the single-level surrogate model. The fourth row in the table lists the optimal design by Daniels (2014), who optimized the original PFT device (the fifth row in the table) by varying one parameter each time. Although this one-factor-at-a-time method improves the power output of the original design from 0.24 to 0.33 by $37.5 \%$, it cannot guarantee a global optimum since the algorithm ignores the coupled effect of two or more factors on the responses of interests. Overall, with the capacity of finding the global optimum design accurately and efficiently, the multi-level surrogate model assisted optimization strategy has improved the power output of the PFT device by $58 \%$ as compared with the original PFT design and 15\% more than the optimal design by Daniels (2014). 
Table 4. Optimal solution with safety factor of 2.0 and original design.

\begin{tabular}{|l|c|c|c|}
\hline \multirow{2}{*}{ Designs } & \multicolumn{2}{|c|}{ Response type } \\
\cline { 2 - 4 } & $\begin{array}{c}\text { Normalized } \\
\text { Electrical power }\end{array}$ & $\begin{array}{c}\text { Normalized maximum } \\
\text { principal stress }\end{array}$ & $\begin{array}{c}\text { Normalized } \\
\text { displacement }\end{array}$ \\
\hline $\begin{array}{l}\text { Predicted by multi-level } \\
\text { surrogate modeling strategy }\end{array}$ & 0.39 & 0.50 & 0.01 \\
\hline $\begin{array}{l}\text { Predicted by single-level } \\
\text { surrogate model (Luo et al., } \\
\text { 2017) }\end{array}$ & 0.46 & 0.50 & 0.01 \\
\hline $\begin{array}{l}\text { Validation by FEA } \\
\text { Optimized design (Daniels, } \\
\text { 2014) }\end{array}$ & 0.33 & 0.49 & 0.009 \\
\hline $\begin{array}{l}\text { Original design (Daniels, } \\
\text { 2014) }\end{array}$ & 0.24 & 0.48 & 0.01 \\
\hline
\end{tabular}

Another observation from the results in Table 2 and Table 4 is that the reduction of power output of the PFT device from 0.99 to 0.38 is not proportional to the increase of the respective safety factor from 1.0 to 2.0 . The effect of the stress safety factor on the maximal power output of optimal designs is given in Table 5. It is noted that when the safety factor is reduced, the electric power output is increased. To achieve the best designs corresponding to the stress safety factor of 1.0, 1.5, and 2.0, respectively, the thickness of the endcap $\left(t_{c}\right)$ and the internal angle $(\theta)$ should be increased gradually, while the thickness of substrate layers $\left(t_{s}\right)$, length of apex $\left(D_{a}\right)$, and length of cavity $\left(D_{c}\right)$ are suggested to take their boundary values. As compared with the original design which has a normalized electric power $(P)$ of 0.33 , the optimal designs obtained by the multi-level surrogate modeling optimization strategy can increase the electric power by 
$15 \%, 76 \%$ and $200 \%$ if the stress safety factor of $2.0,1.5$ and 1.0 are applied, respectively. In Figure 7, a second-order polynomial curve can be determined to best describe the relationship between the power outputs and safety factors of the PFT device. It is concluded that the output power is more sensitive to a lower safety factor, as shown in Figure 7, and using a smaller safety factor will significantly increase the power output of the PFT energy harvester. However, engineering design should consider different levels of safety due to the existence of uncertainties in the real-world, such as variation of geometric parameters and material properties. Ignoring the effect of uncertainties, designs with low safety factors may cause critical failure. In order to achieve a lower safety factor as well as significantly increase the power output of the PFT device, robust optimization of the PFT device under uncertainties aiming at reducing design sensitivity has attracted growing attention from researchers.

Table 5. Optimum Design variables.

\begin{tabular}{|l|c|c|c|c|c|c|l|c|}
\hline $\begin{array}{l}\text { Design } \\
\text { variables }\end{array}$ & $\begin{array}{l}t_{p} \\
(\mathrm{~mm})\end{array}$ & $\begin{array}{l}t_{s} \\
(\mathrm{~mm})\end{array}$ & $\begin{array}{l}t_{c} \\
(\mathrm{~mm})\end{array}$ & $\begin{array}{l}D_{c} \\
(\mathrm{~mm})\end{array}$ & $\begin{array}{l}D_{a} \\
(\mathrm{~mm})\end{array}$ & $\begin{array}{l}\theta \\
(\mathrm{deg})\end{array}$ & $\begin{array}{l}R \\
(\mathrm{M} \Omega)\end{array}$ & $P_{n}$ \\
\hline $\begin{array}{l}\text { Original } \\
\text { design value }\end{array}$ & 4 & 0.6 & 2 & 40 & 14 & 8.75 & 10 & 0.33 \\
\hline $\begin{array}{l}\text { Optimum } \\
\text { value (SF2) }\end{array}$ & 9 & 0.6 & 1.8 & 40 & 10 & 16 & 18.5 & 0.38 \\
\hline $\begin{array}{l}\text { Optimum } \\
\text { value (SF1.5) }\end{array}$ & 7 & 0.6 & 1 & 40 & 9.8 & 14 & 13.4 & 0.58 \\
\hline $\begin{array}{l}\text { Optimum } \\
\text { value (SF1) }\end{array}$ & 7 & 0.6 & 0.92 & 40 & 9.8 & 11.2 & 13.4 & 0.99 \\
\hline
\end{tabular}




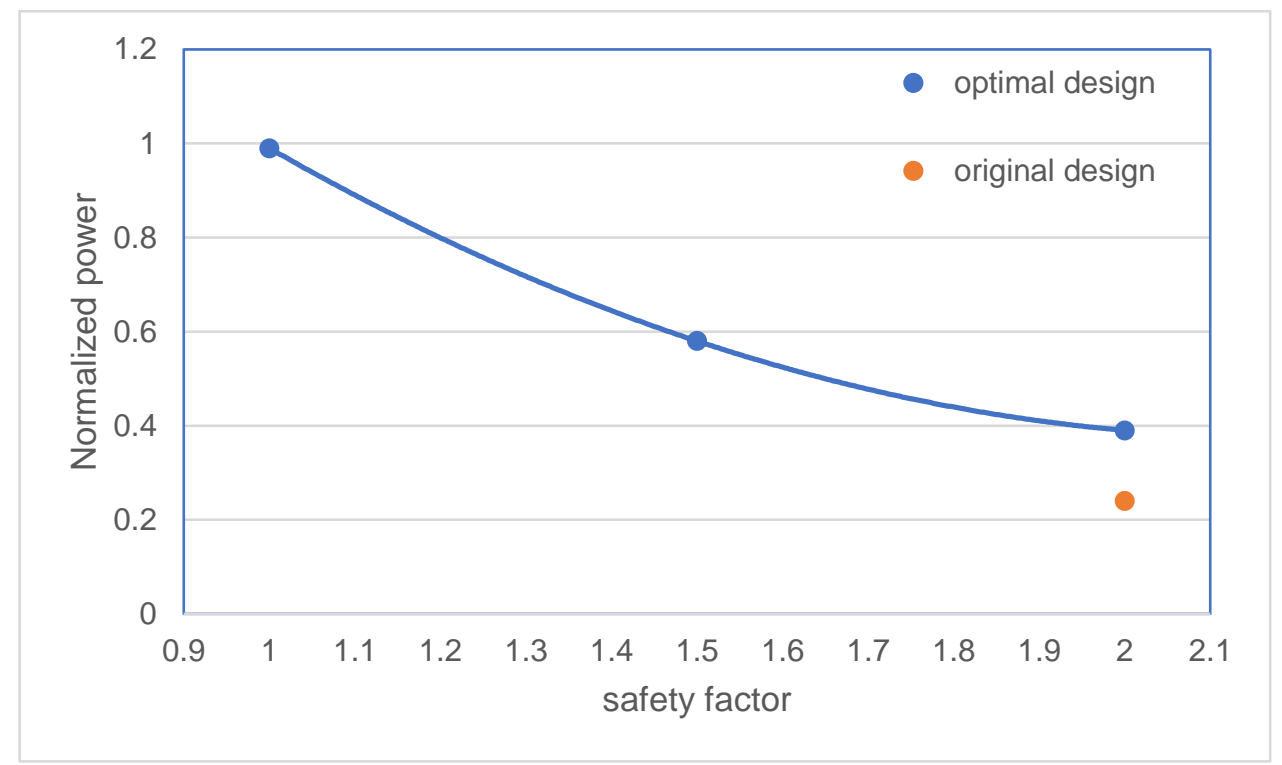

Figure 7. Optimal results of PFT device with different safety factors

\section{Conclusions}

In this paper, a multi-level surrogate modeling strategy for design optimization of a novel PFT device has been proposed to accurately obtain the maximum electric power. An extended Optimal Latin Hypercube (OLH) DoE was further developed to determine sampling points for efficiently building multi-level surrogate models. Based on the near-optimal solution by the global exploration, the maximum electric power with high accuracy was found by local exploitations performed in the vicinity of the refined design domain. Designs with a stress safety factor of $2.0,1.5$, and 1.0 were investigated respectively. Optimization of these designs increased the respective electric power by $15 \%, 76 \%$, and $200 \%$ as compared to the original design of the PFT device.

The results obtained suggest that the proposed multi-level surrogate modeling strategy 
is capable of solving complex design optimization problems more accurately and efficiently than the single-level surrogate modelling approach and the conventional one-factor-at-a-time method. The multi-level surrogate model also provides design engineers with explicit relationships between structural design parameters and energy output of the harvesting devices they are designing.

Future investigations are required on the uncertainty analysis of material properties and damping effects of the PFT device in FE simulations.

\section{Acknowledgements}

The authors are grateful to the EPSRC for financial support (EP/K020080/1).

Declaration of Conflicting Interests The authors declared no potential conflicts of interest with respect to the research, authorship, and/or publication of this article.

\section{References}

Andrade A, Buiochi F, Adamowski J, (2008) Finie Element Analysis of a Piezoelectric Acoustic Levitator, IEEE International Ultrasonics Symposium Proceedings, DOI:10.1109/ULTSYM.2008.0343.

Armani U, Khatir Z, Khan A, Toropov VV, et al. (2011) Control of physical consistency in meta-model building by genetic programming. In: Proceedings of the Second International Conference on Soft Computing Technology in Civil, Structural and Environmental Engineering, Civil-Comp Press, Stirlingshire, UK, Paper 43, 2011. DOI:10.4203/ccp.97.43.

Armani U (2014) Development of a hybrid genetic programming technique for computationally expensive optimisation problems. Dissertation, University of Leeds, UK 
Audze P, Eglais V (1977) New approach for planning out of experiments. Problems of Dynamics and Strengths 35:104-107, Zinatne Publishing House, Riga.

Box JF (1980) R. A. Fisher and the Design of Experiments, 1922-1926, The American Statistician, Vol. 34, No. 1, pp. 1-7. DOI:http://dx.doi.org/10.2307/2682986

Daniels A (2014) Design, analysis and fabrication of a mobile energy harvesting device to scavenge bio-kinetic energy. Dissertation. Cranfield university, UK

Daniels A, Zhu M, Tiwari A (2013) Design, analysis and testing of a piezoelectric flex transducer for harvesting bio-kinetic energy. Journal of Physics: Conference Series 476, 012047. DOI: 10.1088/1742-6596/476/1/012047

Forrester AIJ, Keane AJ (2009) Recent advances in surrogate-based optimization, Progress in Aerospace Sciences 45 (2009) 50-79. DOI: 10.1016/j.paerosci.2008.11.001.

Forrester AIJ, Sóbester A, Keane AJ (2007) Multi-fidelity optimization via surrogate modelling, in: Proceedings of the Royal Society of London A: Mathematical, Physical and Engineering Sciences, vol. 463, The Royal Society, 2007, pp.3251-3269, DOI:10.1098/rspa.2007.1900.

Forrester AIJ, Sóbester A, Keane AJ (2008) Engineering Design via Surrogate Modelling - A Practical Guide. World Health.

Hagood NW, Chung WH, Flotow AV (1990) Modelling of piezoelectric actuator dynamics for active structural control, Journal of Intelligent Material Systems and Structures, (1990) 1, pp.327-354. DOI:https://doi.org/10.1177/1045389X9000100305.

Halder P, Samad A (2017) Torque and efficiency maximization for a wave energy harvesting turbine: an approach to modify multiple design variables, International journal of energy research, 2017, Vol.41(7), p.1014-1028. DOI: 10.1002/er.3694.

Kim J, Park S, Lim W, et al. (2016) Design Optimization of PZT-Based Piezoelectric Cantilever Beam by Using Computational Experiments, Journal of Electronic Materials, Vol. 45, No. 8, 2016 DOI: 10.1007/s11664-016-4497-2.

Koza JR (1992) Genetic Programming: On the Programming of Computers by Means of Natural Selection. MIT press, Cambridge, USA.

Leinonen M, Palosaari J, Juuti J et al. (2014) Combined electrical and electromechanical simulations of a piezoelectric cymbal harvester for energy harvesting from walking, Journal of Intelligent Material Systems and Structures 2014, Vol. 25(4) $391-400$.

Li X, Gao W, Gu L, et al. (2017) A cooperative radial basis function method for 
variable-fidelity surrogate modeling, Struct Multidisc Optim (2017) 56: 1077. DOI: https://doi.org/10.1007/s00158-017-1704-6

Liu B, Koziel S, Zhang QF (2016) A multi-fidelity surrogate-modelassisted evolutionary algorithm for computationally expensive optimization problems, Journal of computational science, Vol.12, p.28-37, DOI: https://doi.org/10.1016/j.jocs.2015.11.004.

Luo L, Liu D, Zhu M and Ye J (2017) Metamodel-assisted design optimization of piezoelectric flex transducer for maximal bio-kinetic energy conversion, Journal of Intelligent Material Systems and Structures 28(18), 2528-2538. DOI: $10.1177 / 1045389 X 17689943$.

Marcelin JL (2004) A metamodel using neural networks and genetic algorithms for an integrated optimal design of mechanisms, Int J Adv Manuf Technol 24: 708-714.

Newnham RE, Zhang J, Meyer R (2000) Cymbal transducers: a review. IEEE Applications of Ferroelectrics, 2000 Page(s): 29 - 32 vol. 1. DOI: 10.1109/ISAF.2000.941506.

Olayan A, Zaki A, Hassan H (2012) Design and Implementation of Thin-filmed Piezoelectric Pressure Sensor, International Journal of Scientific \& Engineering Research, Volume 3, Issue 4, April-2012 1 ISSN 2229-5518.

Poli R, Langdon WB and McPhee NF (2008) A field guide to genetic programming. With contributions by J. R. Koza, Available at: www.gp-field-guide.org.uk.

Siah ES, Sasena M, Volakis JL, et al. (2004) Fast parameter optimization of large scale electromagnetic objects using DIRECT with Kriging metamodeling, ieee transactions on microwave theory and techniques, vol. 52, no. 1, january 2004, DOI:10.1109/TMTT.2003.820891.

Simpson TW, Peplinski JD, Koch PN et al. (1997) On the Use of Statistics in Design and the Implications for Deterministic Computer Experiments, Proceedings of DETC'97 1997 ASME Design Engineering Technical Conferences 1-14.

Sodano HA, Park G, Inman DJ (2004) Estimation of Electric Charge Output for Piezoelectric Energy Harvesting, Strain, Vol.40(2), p.49-58. DOI:10.1111/j.14751305.2004.00120.x.

Sun GY, LI GY, Zhou SW, et al (2011) Multi-fidelity Optimization for Sheet Metal Forming Process, Struct Multidisc Optim 44:111-124, DOI:10.1007/s00158-0100596-5.

Tabatabaei SMK, Behbahani S, Rajaeipour P (2016) Multi-objective shape design optimization of piezoelectric energy harvester using artificial immune system, 
Microsyst Technol (2016) 22:2435-2446, DOI 10.1007/s00542-015-2605-5.

Toropov VV, Bates SJ, Querin OM (2007) Generation of extended uniform Latin hypercube designs of experiments. In: 9th International Conference on the Application of Artificial Intelligence to Civil, Structural and Environmental Engineering, St. Julians, Malta, Civil-Comp Press, Stirlingshire, UK, September, Paper 7, 2007. DOI:10.4203/ccp.87.7

Viana FAC, Haftka RT, Watson LT (2013) Efficient global optimization algorithm assisted by multiple surrogate techniques, J Glob Optim 56:669-689, DOI:10.1007/s10898-012-9892-5.

Vincenzi L, Gambarelli P (2017) A proper infill sampling strategy for improving the speed performance of a Surrogate-Assisted Evolutionary Algorithm, Computers and Structures 178 (2017) 58-70. DOI: 10.1016/j.compstruc.2016.10.004

Yan Z (2018) Modeling of a piezoelectric/piezomagnetic nano energy harvester based on two dimensional theory, Smart Mater. Struct. (2018) 27: 015016 DOI: $10.1088 / 1361-665 X / a a 9 b b d$

Yoon H, Kim M, Park C et al. (2018) Time-varying output performances of piezoelectric vibration energy harvesting under nonstationary random vibrations, Smart Mater. Struct. (2018) 27: 0150004. DOI: 10.1088/1361-665X/aa95ed

Zhu ML, Worthington E, Njuguna J (2009) Analyses of power output of piezoelectric energy-harvesting devices directly connected to a load resistor using a coupled piezoelectric-circuit finite element method, IEEE Ultrasonics, Ferroelectrics, and Frequency Control Society. 2009, Vol.56(7), p.1309-1318. DOI: $\underline{10.1109 / T U F F C .2009 .1187}$ 